\title{
CURSOS DE IFE Y COLABORACIÓN INTERDISCIPLINAR: ROL DE LOS ESPECIALISTAS ${ }^{1}$
}

\author{
Joseba M. González Ardeo²
}

\begin{abstract}
Resumen: El artículo explora y repasa las posibilidades de colaboración entre los profesores de IFE (Inglés para Fines Específicos) y los profesores especialistas de las disciplinas impartidas en ingeniería. El grado de colaboración entre ambos profesionales puede variar enormemente, y va desde el trabajo en equipo hasta la visión individualista carente de sinergia. Otro aspecto considerado en el artículo es cómo afecta esta colaboración al estatus de estos docentes, y qué nuevos enfoques pedagógicos, más eficientes, pueden plantearse ambos. Por último, también se analiza la necesidad que los dos grupos de profesionales tienen el uno del otro.
\end{abstract}

Palabras clave: Colaboración interdisciplinar, IFE, estatus profesional.

Abstract: This paper explores and reviews different possibilities of collaboration between ESP (English for Specific Purposes) specialists, and specialists in disciplines taught in Engineering. The degree of collaboration between both specialists may vary enormously, and it ranges from team-teaching to a synergy-lacking individual approach. Another aspect considered in the paper deals with the way this collaboration affects the status of this part of the teaching body, and what types of more efficient pedagogic approaches both can explore. Finally, the need both specialists have for each other is also analyzed. Key words: Interdisciplinary collaboration, ESP, professional status.

\section{INTRODUCCIÓN}

El IFE (Inglés para Fines Específicos), como asignatura, sigue siendo relativamente desconocido para una buena parte del profesorado universitario de disciplinas técnicas a pesar de que, por un lado, ya desde finales de los 60, como consecuencia del grado de expansión de la actividad científica, técnica y económica, surgió la demanda de un idioma internacional, el inglés, que se convirtió en la llave de la expansión internacional para la tecnología y el comercio y, por otro lado, aunque resulta suficientemente evidente, la transmisión de conocimiento relevante y puntero en el mundo técnico y científico está, casi en exclusiva, irremediablemente vinculado al idioma inglés.

Ya en 1983, Baldauf y Jernudd publicaron que en química, biología, física, medicina y matemáticas, más del 65\% de todas las revistas científicas estaban escritas en inglés. También Swales (1987) apuntaba que más de la mitad de los artículos de investigación publicados a nivel mundial, no necesariamente vinculados a la ciencia y a la tecnología exclusivamente,

$1 \quad$ Fecha de recepción: febrero 2008.

Fecha de aceptación y versión final: junio 2008.

2 Catedrático de Escuela Universitaria, Departamento de Filología y Alemana y de Traducción e Interpretación, Universidad del País Vasco-Euskal Herriko Unibertsitatea; $₫$ fipgoarj@1g.ehu.es. 
se escribían en inglés, cifra que aumentaba espectacularmente si al restringirse a las áreas mencionadas, es decir, química, biología, física, medicina y matemáticas.

Las demandas de la sociedad industrializada trajeron consigo la expansión de un aspecto particular de la enseñanza de la lengua inglesa que no se había dado hasta entonces, a saber, la enseñanza del IFE. Apareció una nueva generación de alumnos, hombres y mujeres de negocios que querían vender sus productos en otros países, técnicos que tenían que leer manuales de instrucciones escritos en inglés, doctores que necesitaban leer revistas especializadas publicadas en inglés o asistir a congresos internacionales para poder estar al día en cuanto a los avances realizados en sus áreas de especialización, en definitiva, toda una variedad de estudiantes cuyos cursos incluían libros de texto y revistas editados sólo en inglés. Es decir, el dominio de esta lengua en determinadas áreas técnicas y científicas pasó a ser un deseo y/o una necesidad.

Más de cuarenta años después de su nacimiento, el IFE ha adquirido una reputación y un reconocimiento que dignifican tanto a quien lo enseña como a quien lo estudia. La razón fundamental para que esto sea así es que se presta una gran atención a las necesidades de los alumnos. En cualquier caso, parece que en el futuro, inmediato, habrá una cada vez mayor demanda de "ingleses" para fines específicos (Dave 2000).

Las célebres palabras de Selinker (1979: 201) "How can we teach the NNS (non-native speaker) to read this stuff when we don't even know what we don't even know?" son un buen argumento y punto de partida para el siguiente apartado.

\section{COLABORACIÓN INTERDISCIPLINAR}

La expresión “colaboración interdisciplinar” (CI) está, en principio, íntimamente ligada al mundo hospitalario y/o sanitario. A nadie se le escapa que, con mucha frecuencia, un diagnóstico de un enfermo en una situación compleja desde el punto de vista de su salud sólo es posible con la colaboración de expertos en distintas especialidades de la medicina y la farmacia. El resultado que se busca de las decisiones adoptadas no es otro que el de curar, o mitigar en lo posible, las dolencias del paciente.

Si extrapolamos esta situación al aula, la pregunta que nos puede surgir de manera inmediata es: ¿Es correcto que cada profesor, encerrado en su burbuja, planifique de manera individual el remedio más adecuado para sus alumnos con la esperanza de que las acciones individuales de cada uno de los profesores implicados en la docencia de esos alumnos den como resultado la formación integral que necesitan? Esta suele ser una situación relativamente habitual en el entorno universitario pero, en el fondo, todos sabemos que la CI puede aportar importantes sinergias.

Con el fin de hacer frente a las necesidades específicas de los alumnos y poder así adoptar una metodología y actividades acordes con el objetivo de la asignatura, el profesor de IFE debe trabajar con los especialistas de los diversos campos. Desafortunadamente y con la excepción de algunos libros de texto diseñados para los campos generales (por ejemplo, computer science o business studies), la mayoría tiende a utilizar temas relacionados con múltiples disciplinas, lo que hace que el material sea a veces repetitivo e incluso confuso para el alumno desde el punto de vista de lo que puede ser apropiado y/o necesario para él. Esta situación lleva a muchos profesores de IFE a la única alternativa que queda: desarrollar 
sus propios materiales. Esto implica una importante labor de investigación por parte del docente, de modo que los materiales finalmente utilizados sean los más apropiados para sus alumnos.

En resumen, la incorporación a la clase de idiomas de material didáctico-educativo, contenido y actividades que se imparten en otros departamentos, así como la participación de profesores de otras áreas, es lo que se conoce como CI en el mundo del IFE.

Por otro lado, si partimos de la base de que la comunicación, o más propiamente dicho, la capacidad para comunicarse, es una competencia clave para los ingenieros, habremos de plantearnos cómo mejorar ésta. Los alumnos, a menudo, no ven relación entre comunicación y el trabajo que desarrollan pues, aparentemente, se limitan a resolver ecuaciones, modelar procesos o llevar a cabo diseño de producto.

Una buena instrucción en comunicación debiera estar firmemente arriostrada y contextualizada en una actividad intelectual auténtica. En definitiva, y como complemento al lenguaje de marcado carácter denotativo del ingeniero, el especialista en IFE debe ofrecer a sus alumnos simulaciones muy cercanas a su entorno profesional y entrenamiento interdisciplinar con un lenguaje de marcado carácter connotativo. Uno de los posibles medios utilizados para conseguir dicha competencia comunicativa de los ingenieros, de manera sinérgica, se asienta en la CI.

\section{VENTAJAS DE LA COLABORACIÓN INTERDISCIPLINAR}

Según diversos autores (Kaufman y Brooks 1996; Master 1997; Peterson 1997), existe poca documentación sobre acciones innovadoras en los programas de preparación de los profesores que estén diseñados para la CI y la integración de idioma y contenido. No obstante, desde el punto de vista del programa de la propia asignatura de "Inglés Técnico", la CI ofrece una mayor flexibilidad al curso habitual de inglés. Además, podemos ampliar el horizonte del curso tradicional de inglés pues se incluyen temas que no se consideran dentro de un programa tradicional de segunda lengua.

En cuanto a las funciones de la universidad, la CI nos permite ser más sensibles a las necesidades sociales y, así, cumplimos uno de los objetivos de la universidad (satisfacer las demandas de la sociedad). Por otro lado, se aporta cohesión y unidad al a menudo fragmentado mundo universitario (departamentos aislados). Además, puede poner fin a la concepción de que los cursos de idiomas en las Escuelas y Facultades de "Ciencias" son impartidos por un departamento "atípico", y puede recolocar a estos docentes en igualdad de condiciones con respecto al resto. Por último, no podemos olvidarnos de que el alumno percibirá que las diferentes asignaturas de su programa de estudios constituyen un entramado real y no están formando parte de compartimentos estancos.

Si consideramos la posible motivación de los estudiantes, creemos que la CI ayuda a responder mejor a las preferencias vocacionales específicas de los estudiantes, así como a sus deseos y necesidades. Debido a su propia naturaleza, la CI convierte a la asignatura en mucho más próxima a los alumnos, y más práctica, ya que los contenidos de otras asignaturas se revisan en la clase de inglés, por lo que tiene más relevancia para la motivación instrumental de los estudiantes. Los temas que se incluyen en los programas, en general, interesan a los alumnos por ser relevantes para ellos y estar familiarizados con el contenido. 
Todos sabemos que la participación directa del alumno en el aula universitaria es, al menos de momento, casi inexistente pues, entre otros motivos, el estudiante tiene, de alguna manera, asumido el rol de agente pasivo, meramente receptor de información. Con la CI, el estudiante puede desarrollar su autoestima pues se da cuenta de que sabe más de lo que creía sobre el tema específico tratado. Es decir, aumenta su confianza para hacer frente, tanto durante como al final de sus estudios, a la bibliografía especializada necesaria para finalizar sus estudios e incorporarse al mercado laboral.

También resulta más gratificante para el profesorado, ya que de este modo sienten que están preparando a sus alumnos para la vida real. Además, la CI es una excelente forma de entrar en el trabajo - la enseñanza- en equipo, tan en boca de los expertos hoy en día. Tampoco podemos olvidarnos de la imagen del personal docente en general y del profesor de IFE en particular pues, con la CI, ésta mejora ya que los alumnos sienten que se trabaja en equipo y, por lo tanto, se está haciendo un esfuerzo real para darles las herramientas que necesitan, y no nos limitamos a seguir un texto convencional que en la mayor parte de los casos no satisface ni sus necesidades ni sus deseos. Por último, el contacto interdisciplinar se podría ver como un posible modelo para el desarrollo del profesor, entendido este como la capacidad para llevar a cabo ajustes de las propias prácticas docentes de acuerdo con las demandas de un programa, las necesidades de los alumnos y del propio centro en el que el profesor enseña.

Otra de las ventajas de la CI es que ésta nos permite enseñar el idioma no como un objeto en sí mismo sino como una especie de vehículo para aprender y comprobar, lo cual de acuerdo con la teoría de Krashen (1985) confirma que el idioma se enseña mejor cuando se utiliza para transmitir mensajes que cuando se enseña como un aprendizaje consciente.

En definitiva, un enfoque de CI (ingeniería, inglés, comunicación) refleja la naturaleza interdependiente del mundo real y empuja a los estudiantes hacia una competencia global que les permitirá interactuar de manera eficiente en diferentes entornos culturales pues esta CI tiene mucho que ver con la comunicación internacional.

A pesar de todas las ventajas apuntadas, también es cierto que un enfoque didáctico fuertemente basado en la CI conlleva una serie de inconvenientes (o meros esfuerzos adicionales) (Castro et al. 1990). De entre ellos, podemos entresacar los siguientes:

- Será necesario llevar a cabo un riguroso análisis para detectar las necesidades de los estudiantes.

- Nos enfrentamos a la apatía y al rechazo de alguno de los profesores con los que es importante colaborar, y nos resultará difícil convencerles de que también ellos pueden conseguir beneficios a través de dicha colaboración (por ejemplo, se pueden revisar y comprobar contenidos en la clase de inglés).

- Tendremos dificultades para obtener material docente apropiado y supondrá un importante trabajo implementar el material existente y diseñar nuevo. Además, tendremos que esforzarnos para adaptar tanto el nivel como los contenidos a nuestra situación.

- Tendremos que transformar la actitud pasiva del estudiante, habitual en el resto de asignaturas que componen su currículo, en una activa, productiva, directa y de implicación personal.

- Habremos de ser especialmente sensibles cuando nos dirigimos a los estudiantes, pues si hacemos evidente el hecho de que tienen una seria falta de conocimientos en su área de 
especialización, esto puede hacer que se salten nuestras clases, especialmente en el caso de estudiantes que muestran rasgos de personalidad característicos de gente introvertida.

- Será necesario un apoyo extra-académico por parte de empresas, instituciones, etc. pues los costes del material a utilizar se disparan (compra de libros, CDs, diccionarios y manuales especializados, etc.).

Como conclusión de este apartado, diremos que el tantas veces mencionado modelo básico de colaboración entre las partes (student, subject teacher, language teacher), hoy en día se ve de manera más compleja y/o completa (ver Fig. 1):

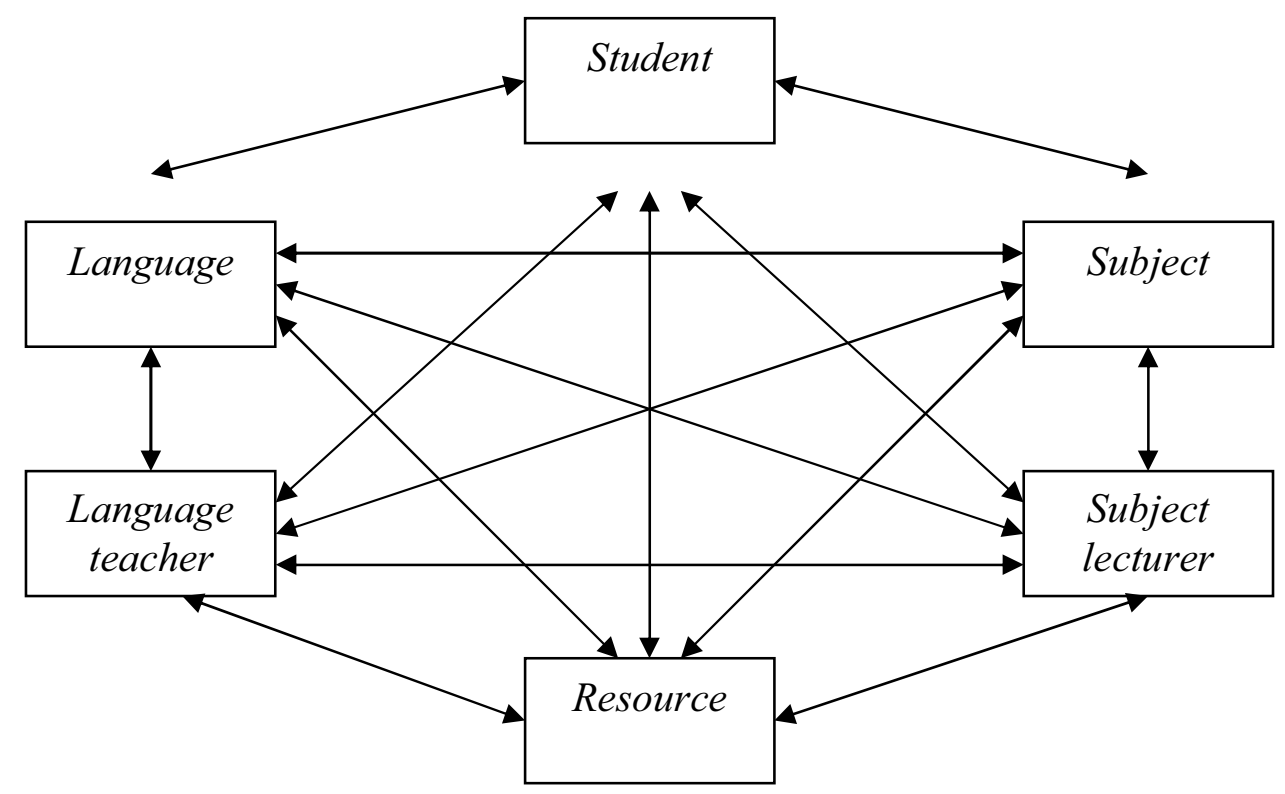

Figura 1. Modelo bidireccional de comunicación interdisciplinar en el IFE

Todo este entramado muestra la lucha continua que el profesor de IFE ha de mantener en aras a conseguir para sus alumnos resultados académicos óptimos pero, muy frecuentemente, algunas de las flechas del diagrama no son bidireccionales (situación óptima), ni tan siquiera unidireccionales.

\section{ESTATUS DEL PROFESORADO IMPLICADO EN COLABORACIÓN INTERDISCI- PLINAR}

El campo del IFE se basa en la colaboración entre un profesional del IFE y los expertos en las materias técnicas correspondientes. El grado de colaboración entre ambos grupos de expertos varía enormemente, pudiéndose considerar el trabajo en equipo el máximo nivel de colaboración posible. La CI afecta al estatus de los profesionales del IFE (Stwart 2001) e influye en la práctica pedagógica.

Son muchos los autores que afirman que el profesor universitario se encuentra más recompensado con la investigación que con la docencia (Sundre 1992; Donnay 1993). Podríamos añadir, por despejar todas las posibles dudas, que dicha recompensa es de carácter pecuniario. De hecho, muchos profesores consideran la enseñanza una interferencia 
en sus trabajos de investigación y sus correspondientes publicaciones. Este planteamiento es relativamente frecuente entre las universidades del área mediterránea, ya que dedicándose a la enseñanza y a los alumnos no se obtiene compensación económica ni prestigio (Ballantine 1989: 294). La conclusión que sacamos es que el estatus social que se le adjudica al profesorado universitario aumenta en prestigio prioritariamente si sus labores de investigación son destacadas. Además, si éstas dan lugar a la concesión de patentes que den lugar a explotación comercial, muy difícil, aunque no imposible, en el campo del IFE, mayor será dicho prestigio. Es evidente que, ante este panorama, el profesorado implicado en el IFE se encuentra a priori en clara desventaja con respecto a sus colegas de áreas técnicas y/o científicas. Algunos autores, por ejemplo Swales (1990), consideran que el uso de métodos de cooperación, como los que analizaremos a continuación, es el primer paso para establecer la paridad con otras disciplinas.

Los programas de doctorado rara o excepcionalmente preparan a los nuevos profesores para las responsabilidades en la enseñanza. La calidad de la enseñanza de un profesor universitario es un factor insignificante a la hora de ascensos, revisiones salariales, etc. De hecho, todos sabemos que a este tipo de llamadas el "docente- investigador" acude fundamentalmente con todo su arsenal investigador para respaldarle y poco o nada puede aportar en el aspecto docente.

Con este panorama, los docentes en su mayoría enseñamos por el método de "ensayo y error". En opinión de Barth (1990), para que tenga lugar un verdadero cambio en la enseñanza, los profesores han de comenzar a ayudarse, además de observar y hablar entre ellos. La enseñanza en equipo es para Buckley (2000: 4) "un grupo de docentes trabajando de manera regular, cooperativa y con unos fines para ayudar a aprender a un grupo de alumnos".

A continuación, vamos a analizar tres opciones diferentes de colaboración interdisciplinar que se dan en contextos geográficos y lingüísticos diferentes.

\section{PRIMER EJEMPLO PRÁCTICO}

Hoy día, la enseñanza del IFE está basada en el siguiente concepto: los estudiantes debieran aprender inglés practicando tareas específicas que les son familiares en su lengua materna. Numerosas investigaciones revelan que aprender un idioma a través de contenido específico es una manera fructífera de aumentar la competencia lingüística (Chuchalin y Danilova 2005).

Un profesor de inglés difícilmente puede ser igualmente profesional en lingüística y en áreas técnicas. Por este motivo, en la Universidad Politécnica de Tomsk (Rusia), se aceptó que el IFE se enseñase, en alianza, por un experto en un área técnica y un instructor de IFE. Este modelo garantiza que el instructor de IFE ayude con temas relacionados con el lenguaje en la dicotomía lenguaje-contenido técnico, mientras que el experto en el área técnica aporta el contenido técnico del curso. Este enfoque colaborativo en el que dos profesores trabajan simultáneamente en el mismo aula recibe el nombre de four-handed instruction (Corin 1997). Es evidente que ambos especialistas han de compartir la misma "longitud de onda" y, así, generar sinergia.

Sin embargo, y a pesar de este planteamiento atípico, la alianza no resultará exitosa ni productiva si el instructor de IFE no tiene un interés genuino por la ciencia y la tecnología 
y el experto en el área técnica no hace nada por adaptar su conocimiento de inglés al nivel adecuado. Además, para ser un buen instructor de IFE, el profesor de idiomas debiera tener un deseo natural por "estar a la última" en cuanto a los últimos descubrimientos en ciencias y tecnología leyendo, observando y escuchando noticias relacionadas con la ciencia.

\section{SEGUNDO EJEMPLO PRÁCTICO}

En la Universidad de Birmingham, un curso de IFE lo da directamente un especialista en la materia técnica correspondiente pero existe, paralelamente, una intensa acción tutorial para hacer frente a los problemas meramente lingüísticos que pueden tener los alumnos. Esta acción tutorial es responsabilidad directa del profesor de idioma (Dudley-Evans 2001). Este enfoque, que para algunos puede resultar novedoso, tiene una antigüedad de más de 20 años. En realidad, Dudley-Evans (2001) se limita a reflejar en su artículo los cambios habidos a lo largo de este periodo en el EAP program. En un primer momento, el trabajo conjunto de profesores de idiomas con profesores especialistas estaba orientado a la ayuda de estudiantes internacionales posgraduados de los departamentos.

Para describir el cambio existente desde esa primera fase, él sugiere una taxonomía de tres niveles de cooperación con el profesorado especialista;

- Primer nivel cooperation $\rightarrow$ El profesor de idioma busca información en el discurso de la comunidad del departamento sobre las expectativas de comunicación en la disciplina por parte de los especialistas, sobre el contenido del curso del especialista y sobre las tareas de evaluación.

- Segundo nivel collaboration $\rightarrow$ Los dos profesores, especialista y del idioma, trabajan juntos fuera del aula para diseñar actividades específicas que son habituales en la asignatura y que ayudan a los alumnos.

- Tercer nivel team teaching $\rightarrow$ El profesor de idioma y el especialista co-enseñan en el mismo aula.

Lo que Dudley-Evans (2001: 226) propone es que nos movamos del nivel cooperation a los niveles superiores para, así, poder desarrollar enfoques que él denomina collaborative y team-taught. En realidad, él se refiere al archifamoso modelo triangular de Swales (1988) en el que se representan las interrelaciones entre los tres participantes en el modelo (alumno, especialista y profesor de idioma), en el que éste último actúa como intermediario entre los otros dos. No obstante, Dudley-Evans (2001: 228) matiza que la clave del éxito para la colaboración en la enseñanza radica en la clara definición de sus roles.

\section{TERCER EJEMPLO PRÁCTICO}

La comprensión del significado de una palabra, una expresión o una oración en su contexto para su utilización posterior de manera productiva será el objetivo último de la actividad aquí planteada.

$\mathrm{La}$ actividad que vamos a presentar resume una experiencia de CI en el campo de la ingeniería química, y más concretamente relacionada con el medio ambiente. Los alumnos de la Escuela Universitaria de Ingeniería Técnica Industrial de Bilbao que cursan estudios conducentes a la obtención del título de Ingeniero Técnico Industrial en Química Industrial 
pueden elegir las asignaturas Inglés Técnico I e Inglés Técnico II. Dentro de esta última, una parte al menos se ha planificado de tal manera que las estructuras lingüísticas de ciertos temas se ven en la clase de Inglés Técnico justo antes de verlas en alguna de sus asignaturas de especialidad. Las fases por las que pasa el alumno son las siguientes:

1. En la clase de Inglés Técnico los alumnos llevan a cabo diferentes tareas escritas y orales destinadas a la adquisición del lenguaje que aparece en el tema y a su correcta pronunciación. Esta tarea se refuerza con otras actividades tales como explicación y/o clasificación de procesos en los que ese lenguaje o esos términos han de aparecer. Aunque no han visto el tema todavía en la asignatura de especialidad, su conocimiento de la ingeniería química y su experiencia les sirven como "pegamento" para poder añadir y asimilar la nueva información.

2. En la clase de la asignatura de especialidad, los alumnos, por ejemplo, visionan un video en inglés sobre el mismo tema, y que incluye el vocabulario recientemente aprendido en la clase de inglés. El profesor detiene el video cada vez que considera que es necesaria alguna aclaración. Las explicaciones las reciben en el idioma utilizado por dicho profesor en su docencia (Euskera o Castellano), entre otras cosas, para asegurarse de que se ha comprendido plenamente.

3. Los alumnos reciben en la clase de Inglés Técnico la transcripción completa del video y realizan diversas tareas con ella, con el fin de fijar las expresiones y comprender las características del texto.

\section{DISCUSIÓN}

En el primero de los casos anteriormente expuestos, vemos que los resultados pueden ser fructíferos pero su puesta en marcha implica que tanto el especialista en IFE como el/los especialista/s en materias técnicas estén presentes simultáneamente en el aula y que haya por parte de ambos una implicación total en el proceso. Es decir, por un lado, el especialista en IFE deberá tener una mentalidad abierta al mundo técnico con toda su complejidad y, por otro lado, el especialista en la materia técnica también deberá tener su mente abierta al planteamiento lingüístico asociado a la CI. El mayor inconveniente que observamos es la dificultad que para un filólogo puede suponer abrir su mente al mundo de la ciencia y la tecnología, opción esta por la que a priori no ha apostado en su vida profesional, muy posiblemente por encontrarse incómodo y/o resultarle difícil su comprensión. Asimismo, el especialista en materias técnicas, difícilmente puede ser simultáneamente especialista en materias lingüísticas y por lo tanto ser capaz de comprender la jerga especializada del filólogo. El caso más extremo pero relativamente habitual es el del especialista técnico que desconoce por completo el inglés y que, por lo tanto, difícilmente podremos colaborar con él.

En definitiva, para que se pueda reproducir el modelo del primer caso analizado se han de cumplir varias condiciones. Por un lado, contar con los recursos humanos necesarios. Por otro lado, y más importante si cabe, que los implicados en la CI estén no sólo animados sino capacitados para poder conseguir el máximo aprovechamiento de la situación.

En cuanto al segundo caso analizado, es evidente que gran número de estudiantes provenientes de todo el mundo optan por realizar sus estudios universitarios en países 
anglosajones y que sus lenguas maternas no son el inglés. Independientemente del nivel de Inglés para Fines Generales con que cuentan dichos alumnos, a la hora de hacer frente a las asignaturas de especialidad en ingeniería, necesitarán ayuda lingüística específica para hacer frente con éxito a sus estudios técnicos. Este es, por tanto, un caso particular que no se reproduce del mismo modo, evidentemente, en países no anglosajones. No obstante, hay que considerar que hace ya muchos años que una corriente procedente del norte de Europa va llegando a nuestras latitudes y es aquí donde podemos encontrar cierto paralelismo. Dicha corriente consiste en ofertar a los alumnos la docencia de una determinada materia en su lengua materna o, alternativamente, en inglés. Es decir, quien opta por la segunda de estas opciones tendrá como profesor que impartir docencia de carácter técnico o científico, en la mayoría de los casos, a un hablante de inglés como segunda o tercera lengua.

En el centro en el que este autor imparte docencia, en breve comenzará a implantarse este nuevo modelo aunque sea de manera muy incipiente. Con la anterior situación, es decir, profesor de IFE y profesor de materia técnica, siempre ha sido el especialista de IFE quien se ha acercado a los especialistas técnicos para buscar y desarrollar la CI. Curiosamente, quien comenzará a poner en marcha el nuevo modelo ya se ha puesto en contacto conmigo para solicitar mi colaboración. Se trata, pues, de una nueva situación que posiblemente nos surja a la mayoría de quienes estamos implicados en el IFE y que será preciso gestionar de manera eficiente.

El tercero de los casos analizados, aquel en el que nos encontramos nosotros inmersos, hace un uso restringido de la CI pero da respuesta a una realidad concreta y, con unos recursos limitados, consigue un excelente aprovechamiento de las oportunidades que nos brinda la CI.

\section{CONCLUSIÓN}

Los niveles de cooperación o CI en el IFE son de diversa naturaleza no sólo dependiendo del país en el que se imparta la asignatura sino de factores tales como el nivel a impartir, los recursos (humanos y técnicos entre otros) disponibles, la predisposición de las partes implicadas (profesor de IFE y profesor o profesores especialistas), etc. En cualquier caso, la CI no es una opción o una posibilidad, sino que creemos que se trata de una obligación.

El nivel de implicación del especialista se puede especificar dependiendo de las circunstancias. En este sentido, podríamos hablar de informador, asesor, consultor, consejero, colaborador, y colega, en el sentido más riguroso y profesional del término. Cualquier nivel de colaboración resulta ser positivo no sólo para los alumnos sino también para el propio profesor de IFE. El IFE, tradicionalmente unido a la ingeniería pero que ha ampliado su horizonte hasta límites insospechados y en las más diversas áreas en los últimos tiempos, sólo podrá alcanzar un estatus similar al de las áreas que pretende complementar (o al menos suplementar), si se da de verdad una imbricación mutua. La consecuencia inmediata, en términos de estatus tanto social como académico, de quien lo enseña es obvia: tenderá a equipararse con el del profesor especialista. Es evidente que otras asignaturas han tenido que luchar anteriormente por conseguir un estatus y un reconocimiento (por ejemplo, la ingeniería, entendida en un sentido amplio, hace mucho tiempo que forjó alianzas con materias firmemente enraizadas y establecidas, tales como las matemáticas, sobre todo, 
y la física). La estrategia a largo plazo de esta batalla ha sido clara, desarrollar la CI por medio de la enseñanza en cooperación o en colaboración, y los resultados conseguidos son también claros, su actual estatus.

En definitiva, en el IFE podemos emular lo que han hecho en ingeniería durante más de un siglo y explotar las relaciones con otros departamentos. Siguiendo este procedimiento, el profesorado de IFE conseguiría:

- mejorar su estatus

- cambiar el rol suplementario del IFE a uno complementario que sirva para que la combinación de IFE y asignaturas de especialización formen una combinación útil y sinérgica

- descubrir, en el sentido dado por Selinker (1979) a sus palabras, qué no sabemos todavía.

\section{REFERENCIAS BIBLIOGRÁFICAS}

BALDAUf, R.B. y R.B. JERNUD. 1983. "Language of publication as a variable in scientific communication". Australian Review of Applied Linguistics 6, 1: 97-108.

Ballantine, J. 1989. "University teaching around the world". Teaching Sociology 17: 291-296.

BARTH, R.S. 1990. Improving Schools from within. San Francisco, CA: Jossey-Bass.

Buckley, F.J. 2000. Team Teaching: What, Why and How? Thousand Oaks, CA: Sage Publications.

Castro, J., C. Ibañez y J. M. GonzÁlez. 1990. “Interdisciplinary Collaboration. The ideal way out in LSP teaching: The fruits of the Bilbao experience." Las Lenguas Aplicadas a las Ciencias. Artículos seleccionados del I Congreso Luso Hispano sobre las lenguas aplicadas a las ciencias. Badajoz (España), 31 Mayo - 2 Junio 1990. Eds. M. Mc. Travers, R. Alejo y S. Gómez. Badajoz: Universidad de Extremadura. 38-43.

Chuchalin, A. I. y E. A. Danilova. 2005. "The Breakthrough of the Internet to Empower ESP Teaching and Learning at Tomsk Polytechnic University". Global Journal of Engineering Education 9, 2: 129-136.

Corin, A, 1997. "A course to convert Czech proficiency to proficiency in Croatian and Serbian". Content Based Instruction in Foreign Language Education: Models and Methods. Eds. S. B. Stryker y B. L. Leaver. Washington, DC: Oryx Press. 78-104.

DAVE, A. 2000. “'Top Ten' significant issues in ELT for the new millennium”. http:/ www1.oup.co.uk/elt/magazine/millennium/authorf/authorf.htlm (20 June 2007).

DonnAY, J. 1993. "Stratégie de formation pédagogique des nouveaux professeurs d'université". Ponencia de "Jornada sobre Formació inicial del Professorat Universitari”. Barcelona: ICE. 
Dudley-Evans, T. 2001. "Team-teaching in EAP: Changes and adaptations in the Birmingham approach". Research Perspectives on English for Academic Purposes. Eds. J. Flowerdew y M. Peacock. Cambridge: Cambridge University Press. 225-238.

Kaufman, D. y J. G. Brooks, 1996. "Interdisciplinary Collaboration in Teacher Education: A Constructivist Approach”. TESOL Quarterly 30: 231-251.

Krashen, S. D. 1985. The natural approach. Oxford: Pergamon Press.

Master, P. 1997. "ESP teacher education in the U.S.". Teacher Education for Language for Specific Purposes. Eds. R. Howard y G. Brown. Clevedon (U.K.): Multilingual Matters.

Peterson, P.W. 1997. "Knowledge, Skills, and Attitudes in Teacher Preparation for Content-based Instruction". The Content-based Classroom: Perspectives on Integrating Language and Content. Eds. M. A. Snow y D. M. Brinton. New York: Longman.

Selinker, L. 1979. "On the Use of Informants in Discourse Analysis for Specialized Purposes". International Review of Applied Linguistics in Language Teaching 17, 3: $189-215$.

STwART, T. 2001. "Raising the Status of ESP Educators Through Integrated Team Teaching”. Asian Journal of English Language Teaching 11: 45-67.

SundRE, D.L. 1992. "The specification of the content domain of faculty scholarship". Research in Higher Education 33, 3: 297-315.

SwaLes, J. 1987. "Utilizing the literatures in teaching the research paper". TESOL Quarterly 21, 1: 48-68. . 1988. Episodes in ESP. New York: Prentice Hall.

. 1990. Genre Analysis: English in Academic and Research Settings. Cambridge: Cambridge University Press. 